\title{
Dynamics of Delay Differential Equations with Its Applications 2014
}

\author{
Chuangxia Huang, ${ }^{1}$ Zhiming Guo, ${ }^{2}$ Zhichun Yang, ${ }^{3}$ Yuming Chen, ${ }^{4}$ and Fenghua Wen ${ }^{5}$ \\ ${ }^{1}$ College of Mathematics and Computing Science, Changsha University of Science and Technology, Changsha 410114, China \\ ${ }^{2}$ School of Mathematics and Information Science, Guangzhou University, Guangzhou 510006, China \\ ${ }^{3}$ College of Mathematics, Chongqing Normal University, Chongqing 400047, China \\ ${ }^{4}$ Department of Mathematics, Wilfrid Laurier University, Waterloo, ON, Canada N2L 3C5 \\ ${ }^{5}$ Business School, Central South University, Changsha 410012, China \\ Correspondence should be addressed to Chuangxia Huang; cxiahuang@126.com
}

Received 11 December 2014; Accepted 11 December 2014

Copyright (C) 2015 Chuangxia Huang et al. This is an open access article distributed under the Creative Commons Attribution License, which permits unrestricted use, distribution, and reproduction in any medium, provided the original work is properly cited.

Delay differential equations have attracted a rapidly growing attention in the field of nonlinear dynamics and have become a powerful tool for investigating the complexities of the real-world problems such as infectious diseases, biotic population, neuronal networks, and even economics and finance. When employing delay differential equations to solve practical problems, it is very crucial to be able to completely characterize the dynamical properties of the delay differential equations. In spite of the amount of published results recently focused on such systems, there remain many challenging open questions.

The aim of this special issue is to gather recent research efforts on the development and applications of delay differential equations and to see the latest developments. This special issue contains twenty-five research articles. The original papers explored in this special issue include a wide variety of topics such as the following.

Asymptotic Analysis and Synchronization. Y. Yuan and Z. Guo proposed a fluctuation method to investigate the global asymptotic stability of a very general class of delayed reactiondiffusion equations. L. Zuo and M. Liu investigated the asymptotical stability for an epidemic model with time delay. Z. Zhang et al. investigated a nonlinear viscoelastic equation with interior time-varying delay and nonlinear dissipative boundary feedback and proved the global existence of weak solutions and asymptotic behavior of the energy by using the
Faedo-Galerkin method and the perturbed energy method. J. Zhang et al. used the Lyapunov function method and Lur'e system approach to study the quasisynchronization in a communication system.

Invariant Sets and Attractor. W. Wu used the theory of exponential dichotomy on time scales and fixed point theory based on monotone operator to the global attractivity of the almost periodic solution for a predator-prey system with Beddington-DeAngelis functional response on time scales.

Stability Analysis. P. Wang et al. established a criterion on integral $\varphi_{0}$-stability in terms of two measures for impulsive differential equations with "supremum" by using the conevalued piecewise continuous Lyapunov functions, Razumikhin method, and comparative method. C. Liu and Y. Li investigated the global stability of a nonautonomous twospecies competitive system with stage structure and double time delays. L. He and X. Wang proposed a novel method for studying the stability of a macroeconomic system with fractional derivative. H. Peng and Z. Guo proposed a viral infection model with delay and obtained some necessary and sufficient conditions to ensure the global stability of the model.

Bifurcation Analysis. Y. Zhai et al. investigated an avian influenza virus propagation model with nonlinear incidence 
rate and delay based on SIR epidemic model and established the global existence of periodic solutions by using a global Hopf bifurcation theory. Y. Dai et al. investigated the local stability of the equilibria and the existence of Hopf bifurcation for a predator-prey system with Michaelis-Menten type functional response and two delays.

Oscillation and Boundary Value Analysis. S. Guo et al. were concerned with oscillation of the first order neutral delay differential equation with constant coefficients and obtained some necessary and sufficient conditions of oscillation for all the solutions in respective cases. Z. Ouyang and H. Liu investigated a class of fractional order three-point boundary value system with resonance. J. Liu and L. Yan used the variational method to investigate the solutions of damped impulsive differential equations with mixed boundary conditions.

Periodic Solutions Analysis. R. Hu used the LyapunovSchmidt reduction method and computations of critical groups to study a higher order difference equation and proved that the equation has four $M$-periodic solutions. G. Lin and Z. Zhou used the critical point theory to obtain a new sufficient condition on the existence of homoclinic solutions of a class of nonperiodic discrete nonlinear systems in infinite lattices. T. Zhang et al. investigated the existence and global attractivity of the "infection-free" periodic solution for a new epidemic disease model governed by system of impulsive delay differential equations.

Numerical Computation Analysis. D. Olvera et al. expanded the application of the enhanced multistage homotopy perturbation method (EMHPM) to solve delay differential equations (DDEs) with constant and variable coefficients.

The response to this special issue was beyond our expectation. We received 46 papers in the interdisciplinary research fields. This special issue includes twenty-five high-quality peer-reviewed papers. These papers contain several new, novel, and innovative techniques and ideas that may stimulate further research in every branch of pure and applied sciences.

\section{Acknowledgments}

The authors would like to express their deepest gratitude to the reviewers, whose professional comments and valuable suggestions guaranteed the high quality of these selected papers. The editors would like to express their gratitude to the authors for their interesting and novel contributions. They would also like to thank the editorial board's members of this journal, for their support and help throughout the preparation of this special issue. The interested readers are advised to explore these interesting and fascinating fields further. The authors hope that problems discussed and investigated in this issue may inspire and motivate discovery of new, innovative, and novel applications in all areas of delay differential equations. Chuangxia Huang would like to express the gratitude to the support of the National Natural Science Foundation of China (nos. 11101053 and 71471020), the Key Project of the Chinese Ministry of Education (no.
211118), and the China Postdoctoral Science Foundation (no. 20140550097).

Chuangxia Huang Zhiming Guo Zhichun Yang Yuming Chen Fenghua Wen 


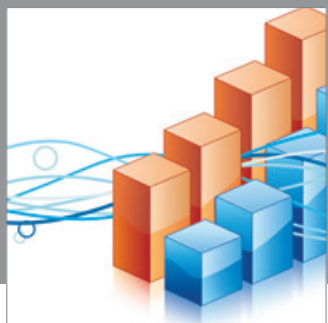

Advances in

Operations Research

mansans

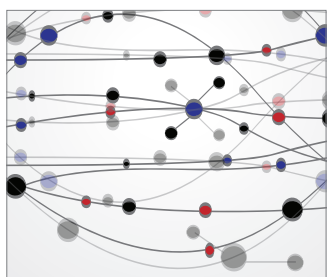

The Scientific World Journal
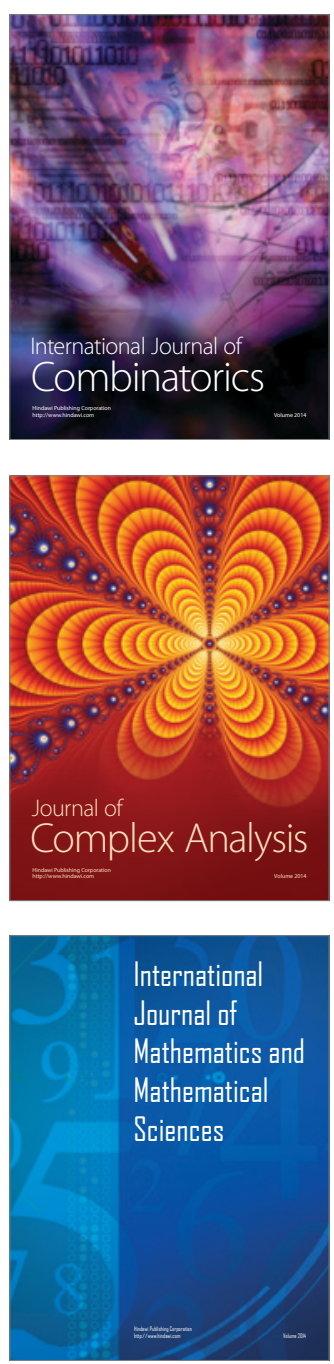
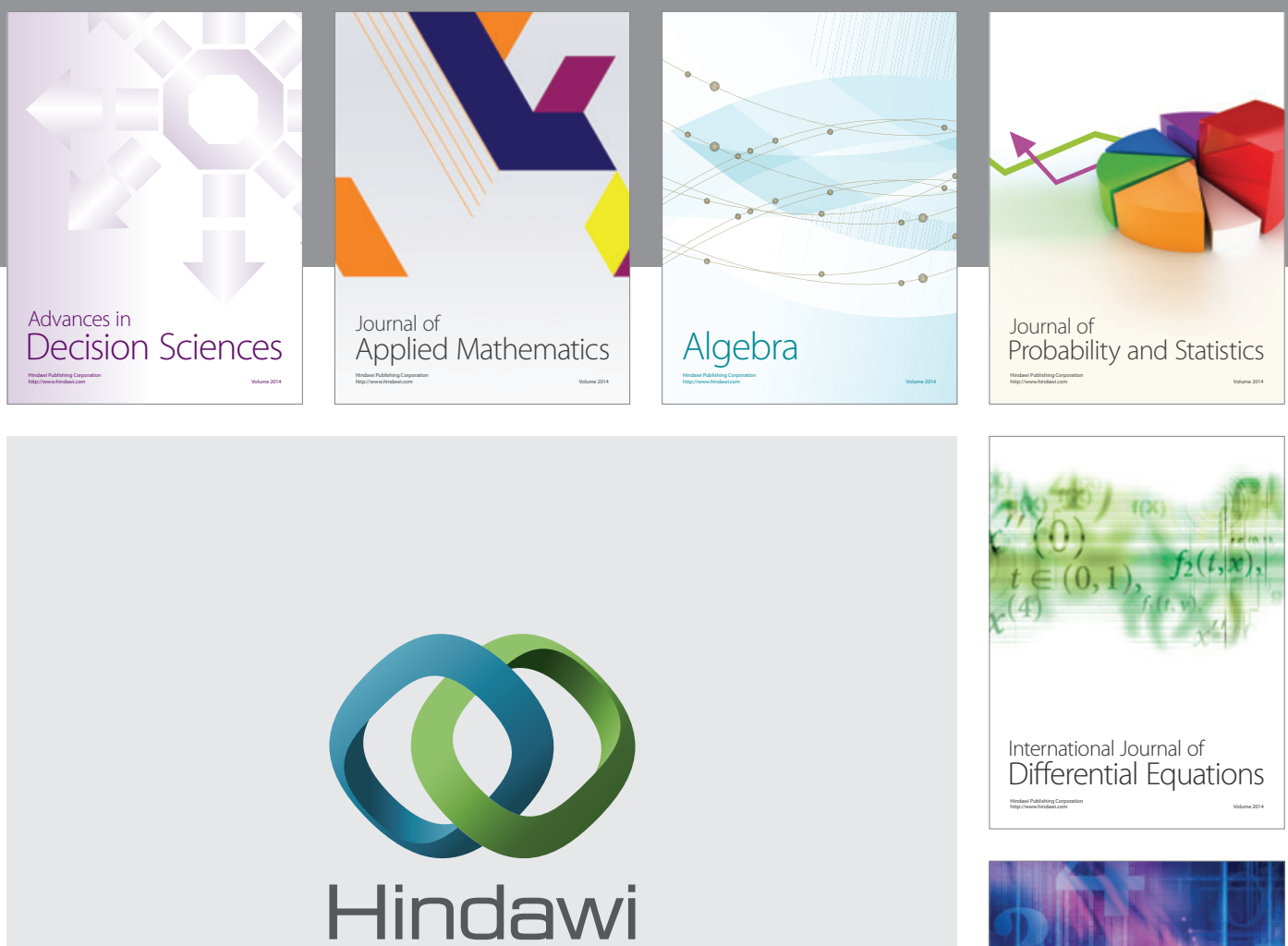

Submit your manuscripts at http://www.hindawi.com
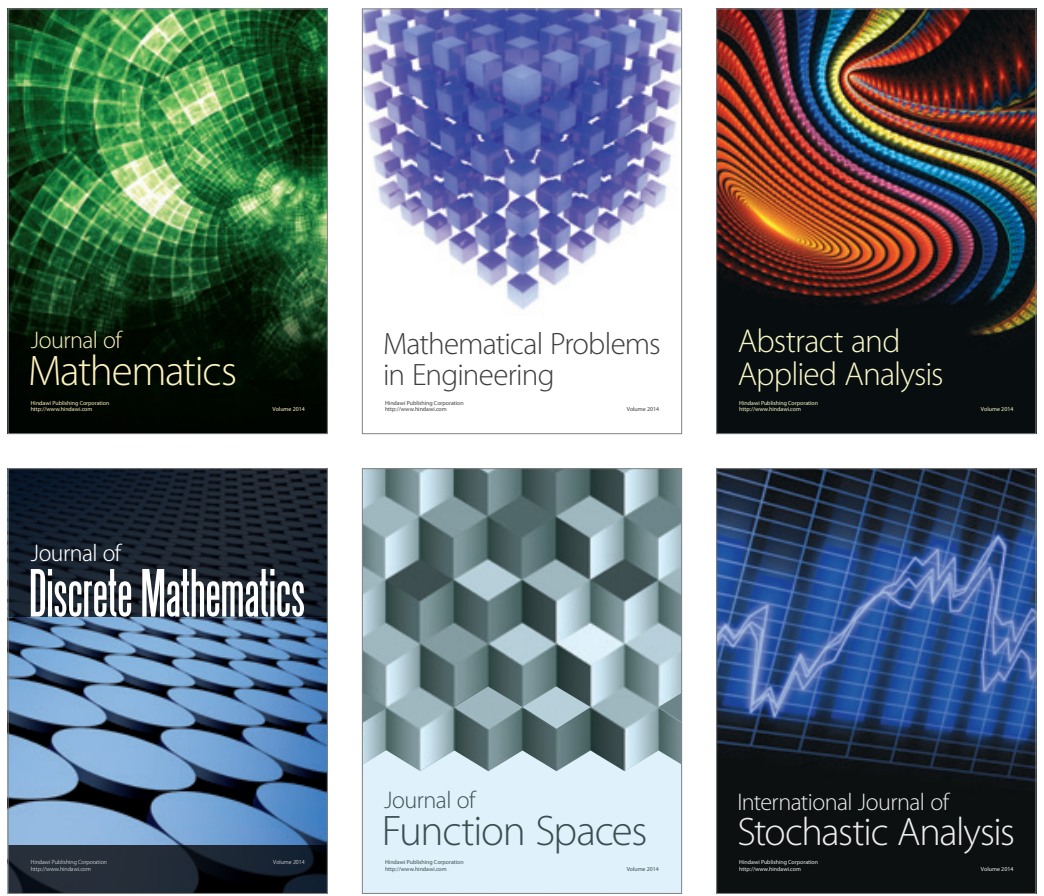

Journal of

Function Spaces

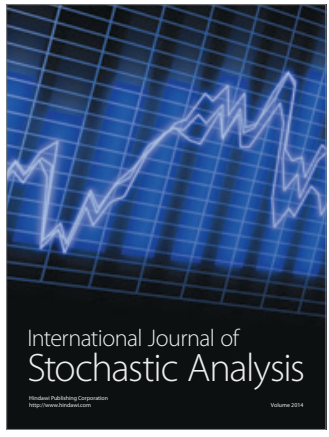

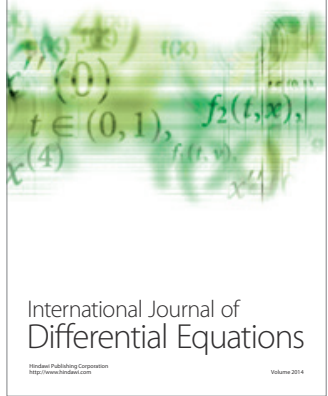
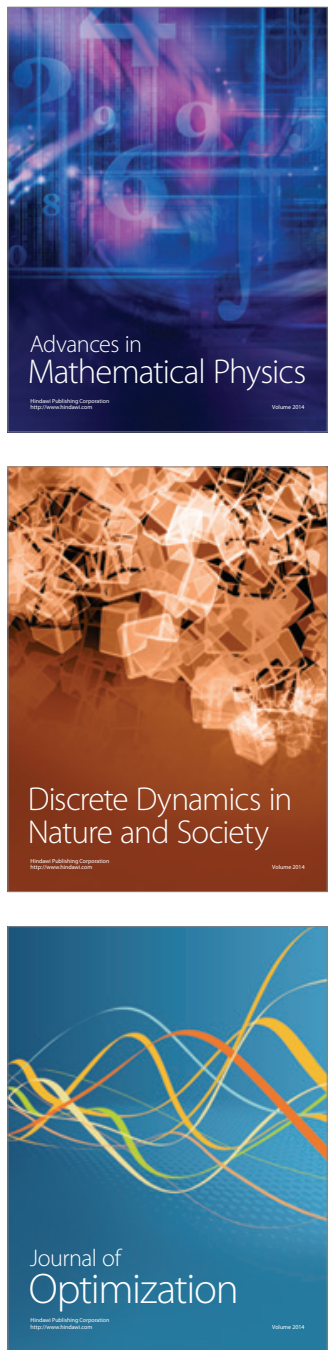